\title{
Consequências da subnotificação dos casos de COVID-19 para a saúde pública no Brasil
}

\section{Consequences of underreporting cases of COVID-19 for public health in Brazil}

\author{
Tiago Mendonça de Oliveira ${ }^{1}$ \\ Ana Carolina Oliveira Araújo ${ }^{2}$
}

\section{RESUMO}

Objetivou-se nesta carta discutir a importância da detecção dos casos de COVID-19 para fomentar ações e estratégias de controle. Observou-se uma baixa realização de diagnósticos no sistema de saúde brasileiro. Essa situação traz algumas consequências negativas para o sistema de saúde, tais como: a persistência da doença, a sobrecarga nos serviços hospitalares, o aumento das repercussões graves e muito graves e a dificuldade de controle da transmissão de COVID-19. Alguns dados foram analisados, como o percentual de testagem no Brasil e a evolução dos casos conforme as regiões brasileiras. A baixa testagem e a dificuldade de diagnostico tem contribuído para a persistência de novos casos no Brasil. Além disso, este relato reforça a importância de se investir no isolamento e distanciamento social e na diminuição do deslocamento das pessoas, como medidas de redução de casos novos.

Termos de indexação: testagem, subnotificação, COVID-19, distanciamento social

Uma doença de caráter respiratório iniciou-se em dezembro de 2019 na China na província de Hubei na cidade de Wuhan e se espalhou rapidamente por todo país e para outras partes do mundo [1]. Dados do dia 16 de março de 2020 [2] demonstraram um total de infecções próximo a 167,5 mil casos, com 13,9 mil novos casos e 6,6 mil mortes causados pelo COVID-19. No entanto, estes números crescem a cada dia. No relatório do dia 26 de março de 2020 tínhamos 462,6 mil casos, com 49,2 mil novos casos e 20,8 mil mortes causados por COVID-19 [3]. Cinco meses após o surgimento do coronavírus, chegamos a uma marca de 823,6 mil casos confirmados, 72,7 mil novos casos e 40,5 mil mortes causados pelo vírus [4].
No Brasil, foram confirmados 904 casos até o dia 20 de março de 2020 em 24 estados e no Distrito Federal. Sete dias após (26 de março), foram registrados 2915 casos confirmados, chegando a 6.836 casos no dia 01 de abril de 2020. Além disso, foram registrados 11 óbitos no dia 20 de março de 2020, 77 óbitos no dia 26 de março de 2020 em oito estados brasileiros e sete dias após passamos para 241 óbitos em 14 estados brasileiros [5,6].

Os dados de COVID-19 cresceram de forma exponencial em algumas partes da Europa e das Américas. A figura 1 justifica a situação brasileira segundo as regiões brasileiras no período 20 de março até 11 de abril de 2020.

${ }^{1}$ Departamento de Medicina Veterinária Preventiva/Epidemiologia da Escola deVeterinária, Universidade Federal de Minas Gerais, Instituto Federal Goiano - Campus Ceres, Goiás, Brasil. Avenida Presidente Antônio Carlos 6627, Campus da UFMG, Cx. Postal 567, Belo Horizonte, Minas Gerais, 30123-970, Brasil. Autor de correspondência: tiago0725@gmail.com

${ }^{2}$ Faculdade de Odontologia da Universidade Federal de Alfenas, Minas Gerais, Brasil.

Como citar este artigo / How to cite this article

Oliveira TM, Araujo ACO. Consequências da subnotificação dos casos de COVID-19 para a saúde pública no Brasil. InterAm J Med Health 2020;3:e202003062. 


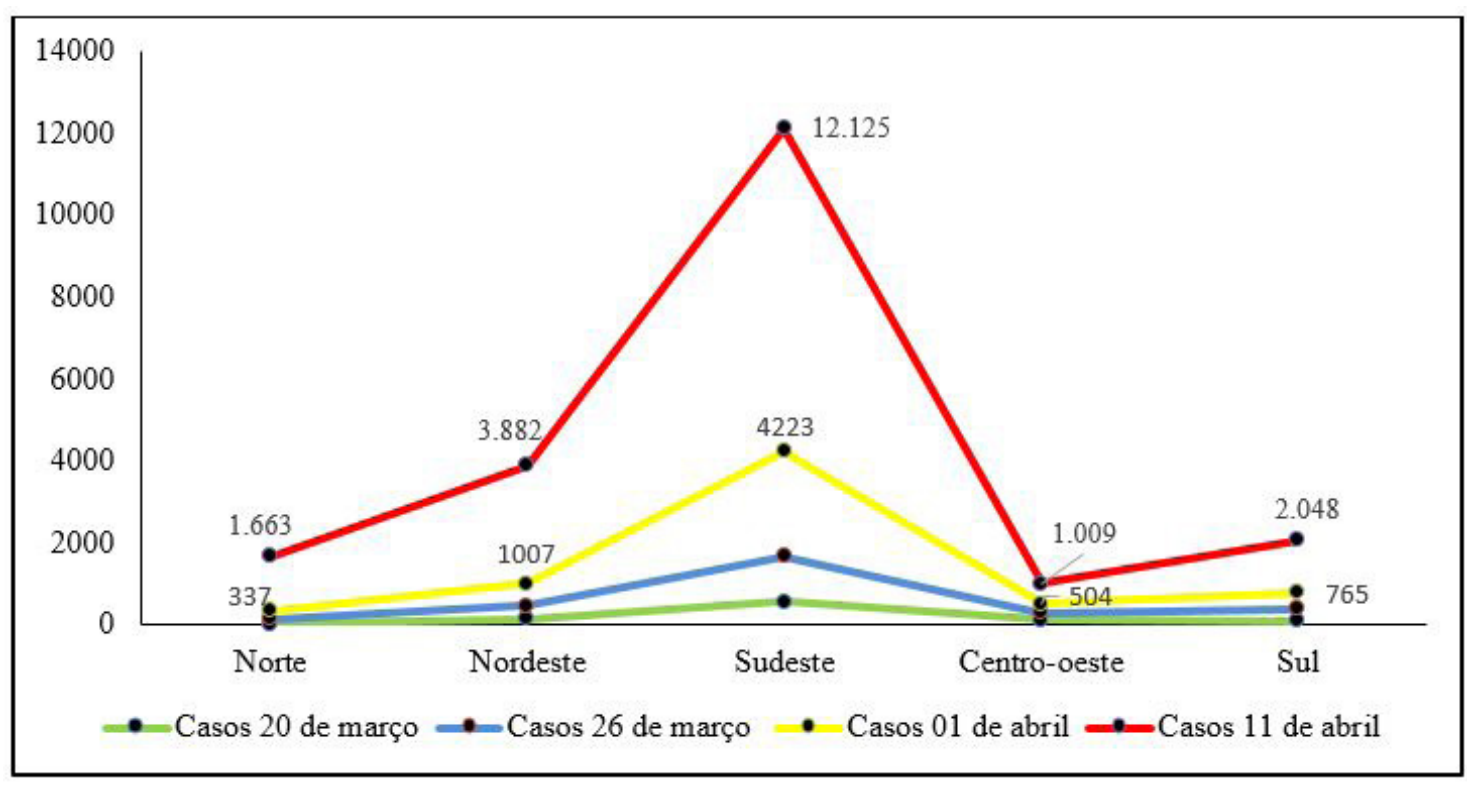

Figura 1. Distribuição de casos confirmados de COVID-19 entre 20 de março e 11 de abril de 2020. Fonte: Ministério da Saúde [5,6].

Com o avanço da COVID-19 espera-se que o número de infecções cresça a cada dia, devido às diversas condições epidemiológicas, como: as características de transmissibilidade da doença e de situações especiais como a presença de pessoas assintomáticas para COVID-19. Essas condições aliadas à dificuldade de testagem diagnóstica em massa podem contribuir para a dispersão do vírus.

Na figura 2 foi demonstrado o número de testes realizados por país por milhão de pessoas, segundo o site Worldometers [7]. Observou-se que dentre os países selecionados a Colômbia e o Brasil fizeram menos de 1.000 testes por milhão de pessoas entre até dia 12 de abril de 2020. Estados Unidos da América (535,1 mil), Espanha (166 mil), Itália (153,2 mil), França (129,6 mil) e Alemanha (125,9 mil) foram países com maiores números de casos confirmados até a mesma data. O Brasil ocupava a $14^{\circ}$ posição do ranking de casos confirmados, com 21.042 casos até dia 12 de abril de 2020. Os demais países desse ranking possuíam menos de 11.000 casos confirmados, segundo o site Worldometers [7].

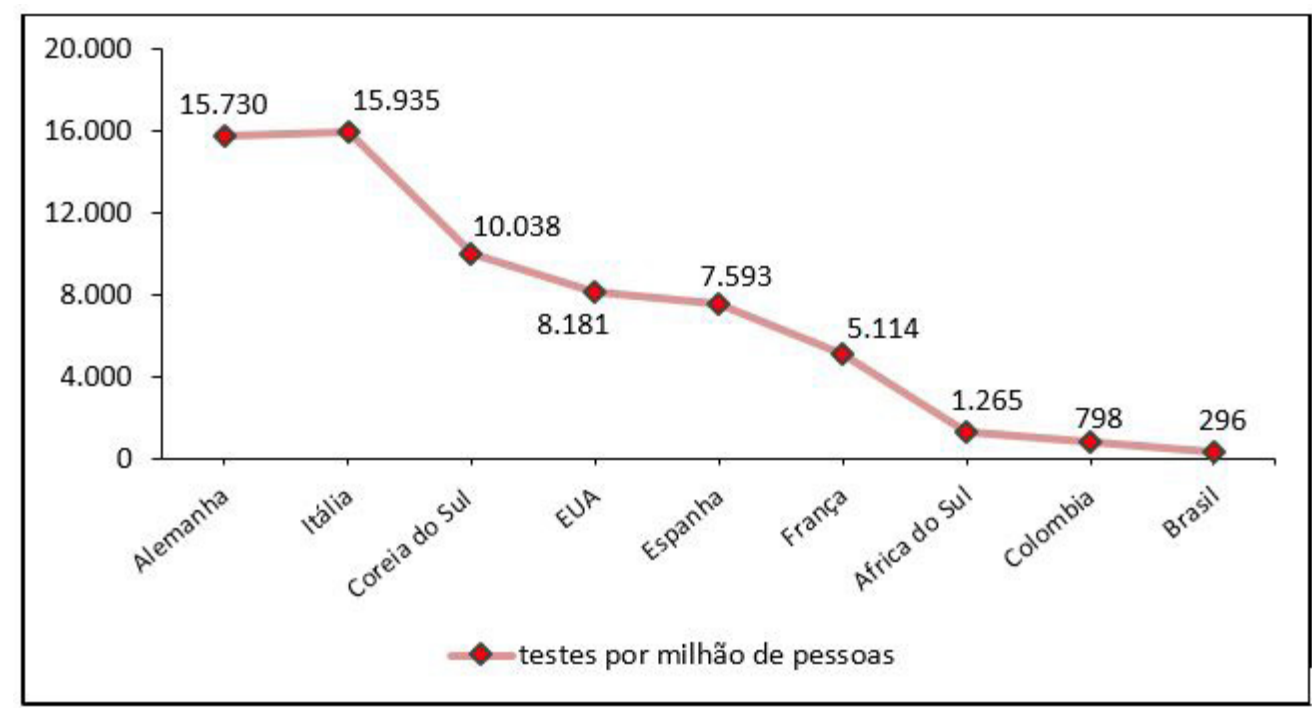

Figura 2. Dados do número de testes de COVID-19 por milhão de pessoas em 12 de abril. Dados coletados no site https://www.worldometers.info/coronavirus/ em relatórios oficiais dos países. 
A recomendação da Organização Mundial da Saúde (OMS) [8] foi a testagem em massa nos diversos países, pois quanto mais diagnósticos assertivos, maiores seriam as condições dos agentes de saúde em discutir e atuar nos determinantes epidemiológicos do COVID-19, além de auxiliar na tomada de decisão. Países como Coreia do Sul e Alemanha tiveram êxitos no controle de COVID-19, devido ao elevado número de testes diagnósticos realizados, ao isolamento social e pela busca ativa dos casos confirmados. A detecção da infecção precocemente ou mesmo em pacientes mais jovens e/ou assintomáticos diminuiria 0 risco da infecção e quebraria a cadeia de transmissão. No entanto, observou-se uma dificuldade de testagem em alguns países principalmente pela falta de insumos para a realização de testes moleculares e pela falta de testes rápidos. Como a demanda mundial por testes rápidos foi muito alta, alguns países testaram apenas casos especiais, dessa forma, essa condição poderia contribuir para a subnotificação dos casos de COVID-19.

O Centro para Modelagem Matemática de Doenças Infecciosas da Escola de Medicina Tropical de Londres [9], do Reino Unido calculou que o Brasil teria aproximadamente mais de 15 mil casos de coronavírus - cerca de 11 vezes mais casos do que os registrados oficialmente, com uma taxa detecção de $11 \%$. Gaete [10] estimou um número de infectados no Brasil em 312.288 até o dia 11 de abril de 2020. Já o Ministério da Saúde (MS), não conseguiu estimar a taxa de subnotificação dos casos brasileiros. Li et al. 2020 [11], estimaram através de modelagens que $86 \%$ de todas as infecções não foram documentadas na China.

Essa subnotificação dos casos é causada por alguns fatores, como a falta de testes de diagnósticos, em um cenário em que apenas pacientes com quadros sintomáticos e manifestações graves da doença são priorizados para testagem. Além disso, os casos assintomáticos de COVID-19 acabam passando despercebidos pelo sistema de saúde, não sendo contabilizados pelos órgãos competentes. Como consequências teremos a persistência da doença e a sobrecarga nos serviços de saúde, por isso é necessário aumentar a sensibilidade de detecção de casos assintomáticos e sintomáticos diminuindo as possibilidades de colapsos nos sistemas hospitalares no Brasil e no mundo. Os desafios na realização de diagnósticos precoces podem atrasar na implementação de medidas de controle e implicar em repercussões muito graves, como o aumento do número de óbitos e o crescimento substancial da dificuldade em restringir o avanço da transmissão de COVID-19. Nesse sentido, abandonar o isolamento social sem ter uma dimensão mais realista do número de casos de COVID-19, poderia colocar a população mais vulnerável em risco, como idosos, imunossuprimidos, portadores de comorbidades e pessoas de baixa renda.

Com base nessas informações fica claro que é preciso investir no isolamento e distanciamento social e na diminuição do deslocamento das pessoas para auxiliar na redução de novos casos $[12,13]$. Além disso, é preciso aumentar a testagem dos casos de COVID-19 para aprimorar a sensibilidade da vigilância em saúde, a fim de melhor direcionar as ações e delinear novas estratégias de controle da doença no Brasil e no mundo.

\section{REFERÊNCIAS}

1. Xiang $Y T$, Yang $Y$, Li W, Zhang L, Zhang Q, Cheung T, et al. Timely mental health care for the 2019 novel coronavirus outbreak is urgently needed. Lancet Psychiatry. 2020; 7: 228229. http://dx.doi.org/10.1016/S2215-0366(20)30046-8

2. World Health Organization, Coronavirus disease (COVID-2019) situation reports 56; Disponível em: $<$ https://www.who.int/docs/default-source/coronaviruse/ situation-reports/20200316-sitrep-56-covid-19. pdf?sfvrsn=9fda7db2_2> Acesso em 16 de março de 2020.

3. World Health Organization, Coronavirus disease (COVID-2019) situation reports 66; Disponível em: $<$ https://www.who.int/docs/default-source/coronaviruse/ situation-reports/20200326-sitrep-66-covid-19. pdf?sfvrsn=81b94e61_2> Acesso em 26 de março de 2020.

4. World Health Organization, Coronavirus disease (COVID-2019) situation reports 72; Disponível em: $<$ https://www.who.int/docs/default-source/coronaviruse/ situation-reports/20200401-sitrep-72-covid-19. pdf?sfvrsn=3dd8971b_2> Acesso em 01 de abril de 2020.

5. Brasil, Ministério da Saúde (『2020)凹 - Disponível

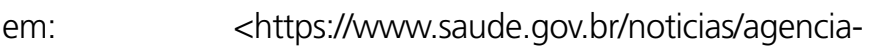
saude/46568-ministerio-da-saude-declara-transmissaocomunitaria-nacional> Acesso em 26 de março de 2020.

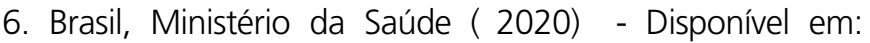
<https://covid.saude.gov.br/> Acesso em 11 de abril de 2020.

7. Worldometers ( $₫ 2020) \rrbracket$ - Disponível em: < https://www. worldometers.info/coronavirus/>Acessoem12deabrilde2020. 
8. World Health Organization. ( $₫ 2020)$ \. Laboratory testing strategy recommendations for COVID-19: interim guidance, 21 March 2020. World Health Organization. License: CC BY-NC-SA 3.0 IGO Disponível em: <https://apps.who.int/iris/ handle/10665/331509> Acesso em 27 de março de 2020.

9. Russell TW, Hellewell J, Abbott S, Jarvis $\mathrm{Cl}$, Zandvoort $\mathrm{KV}$, Flasche $\mathrm{S}$, et al. Using a delay-adjusted case fatality ratio to estimate under-reporting Disponível em: < https://cmmid.github.io/topics/covid19/severity/_global_ cfr_estimates.html> Acesso em 25 de março de 2020.

10. Gaete, R. (2020) Disponível em: < https://ciis.fmrp.usp.br/ covid19/analise-subnotificacao/>Acessoem12deabrilde2020.

11. Li R, Pei $S$, Chen $B$, Song $Y$, Zhang $T$, Yang $W$, et al. Substantial undocumented infection facilitates the rapid dissemination of novel coronavirus (SARS-CoV2). Science. 2020. http://dx.doi.org/10.1126/science.abb3221

12. Moritz UG Kraemer MUG; Chia-Hung Y; Gutierrez B; Wu $\mathrm{CH}$; Klein $\mathrm{B}$; Pigott DM; et al. The effect of human mobility and control measures on the COVID-19 epidemic in China. Science. 2020. http://dx.doi.org/10.1126/science.abb4218

13. Oliveira TM. Manifestações e aglomerações em períodos de pandemia por COVID-19. IJAMH. 2020 . http://dx.doi.org/10.31005/iajmh.v3i0.109 